\title{
NGC 2660 revisited
}

\author{
T.E. Mølholt ${ }^{1}$, S. Frandsen ${ }^{1}$, F. Grundahll ${ }^{1,2}$ and L. Glowienka ${ }^{1}$ \\ ${ }^{1}$ Dept. of Physics and Astronomy, University of Aarhus, Ny Munkegade, Bygn. \\ 1520, DK 8000 Aarhus C, Denmark \\ 2 Danish AsteroSeismology Centre, University of Aarhus, Ny Munkegade, Bygn. \\ 1520, DK 8000 Aarhus C, Denmark
}

\begin{abstract}
A new search for variable stars in NGC 2660 has revealed a number of interesting targets. The cluster has more $\delta$ Scuti members than seen before and an eclipsing binary, where one of the components may be a variable star too.
\end{abstract}

\section{Introduction}

Open clusters as well as globular clusters are becoming increasingly important for the test of stellar evolution and for studies of individual stars of special importance, where one can use the membership to infer some of the basic properties of the star (age, metal content etc.). Some years ago we initiated a programme to search for $\delta$ Scuti stars in open clusters, which together with other similar searches have produced a list of clusters with many $\delta$ Scuti star members. The philosophy has been, that by analysing many variables at the same time, we could learn more about the individual stars. Due to the very difficult mode identification problem, this has turned out not to provide the answers straight away.

Examples from this programme on clusters are the first observations of NGC 2660 (Frandsen et al. 1989), a campaign on NGC 6134 (Frandsen et al. 1996), variables in NGC 1817 (Arentoft et al. 2005) and NGC 7062 (Freyhammer \& Arentoft 2001). NGC 1817 is a particularly interesting case with the largest set of $\delta$ Scuti stars of any cluster. A new cluster added to the set is NGC 2506, which is presently being analysed. Finally, one should also mention the work on nearby clusters (Praesepe and the Pleiades, e.g., Fox Machado et al. 2006).

We would very much like to get the mass of at least one $\delta$ Scuti star by comparing observed frequencies with model frequencies. Then the mass of 
other stars in the cluster could be inferred from the position on the isochrone in the HR diagram. Unfortunately, we have not been able to match theoretical and observed frequencies very well so far, and no accurate masses have been determined.

Another possibility to get the mass is to locate detached eclipsing binaries in clusters and use these systems to derive accurate masses. Again, we can then use the isochrone to derive masses for other stars, in particular the $\delta$ Scuti stars. This will make the comparison of model frequencies and observed frequencies considerably easier. Using large telescopes/efficient spectrographs the observations are now of a quality, which even for faint stars in clusters lead to accuracies of less than $1 \%$ in mass and radius.

\section{The cluster and the observations}

NGC 2660 has an age $t \sim 1$ Gy, a distance module of $(m-M)_{0}=12.2$ corresponding to a distance of $D=2750 \mathrm{pc}$ and a reddening $E(B-V)=0.4$. The metal content is solar: $[\mathrm{Fe} / \mathrm{H}] \approx 0.0$. The cluster has been observed by Frandsen et al. (1989) and Sandrelli et al. (1999)

During the period Feb. 5 to Feb. 272005 new data was obtained with the Danish $1.54 \mathrm{~m}$ telescope at La Silla during time, where the main targets could not be observed. About 1500 frames in the filters Bessel B (464) and Gunn I (949) were acquired. A small set (45) of $V$ frames were observed as well. The camera has a field of view of $13.7 \times 13.7$ square minutes and exposure times were between $60-120 \mathrm{~s}$ in $\mathrm{B}$ and $20-60 \mathrm{~s}$ in I. The $\mathrm{V}$ frames are only used to calculate colours and are not part of the time series analysis.

The reduction was done with the DAOPHOT/ALLSTAR (Stetson 1987) code.

\section{Results}

A search for variables in the combined set of old and new data confirms the presence of several $\delta$ Scuti stars and a detached eclipsing binary system close to the cluster Colour-Magnitude turnoff. Some of the variables are indicated in Fig. 1. There is clearly a set of variables above the turnoff, that one would expect to show pulsations on time scales of hours as they are situated in the instability strip.

The variable stars that might be members of the clusters based on the distance to the cluster center and the magnitude are listed in Table 1 . The magnitude and colour are only indicative, as they have been derived from different datasets. Therefore no errors are given. The pulsation properties are given in Table 2. 


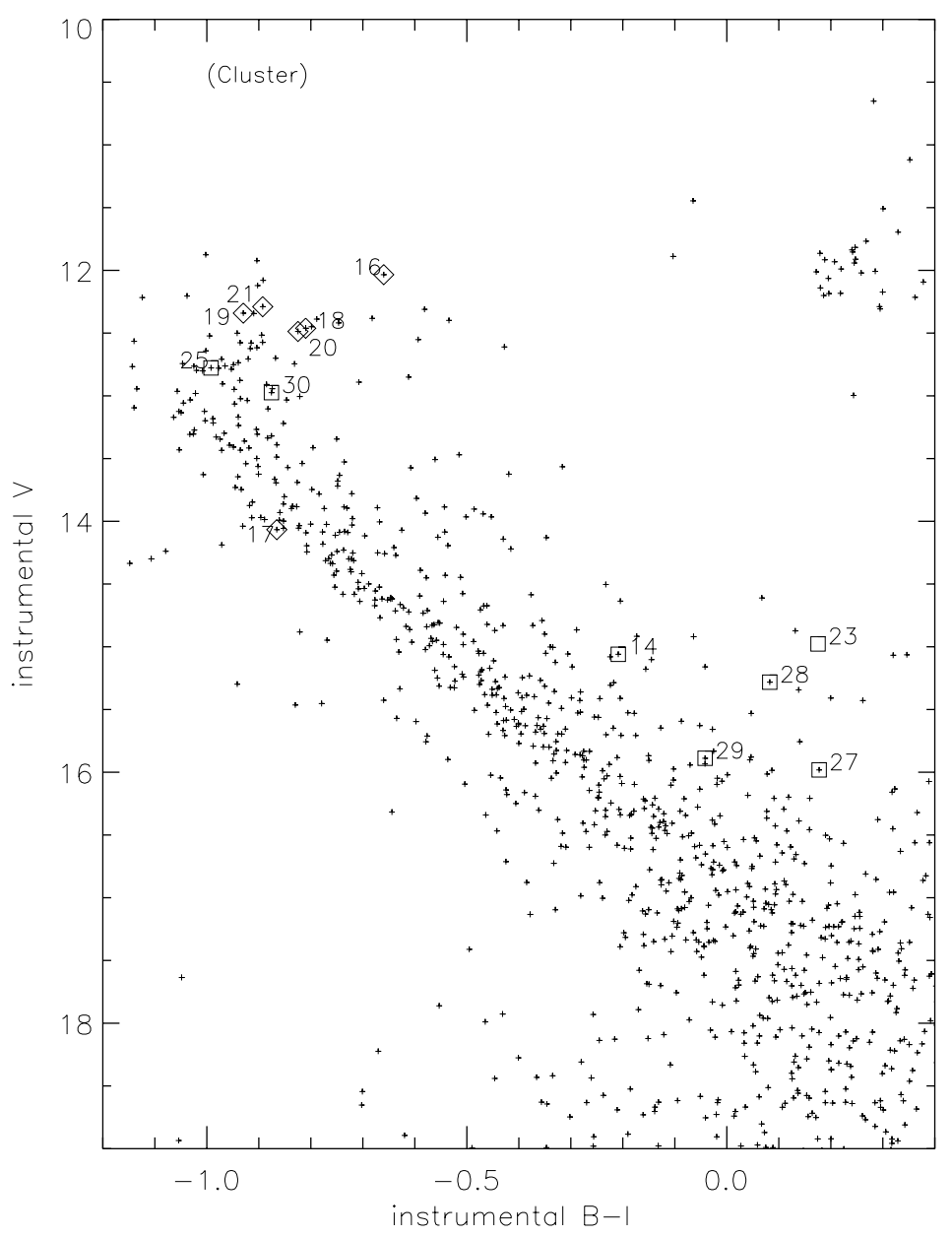

Figure 1: CM diagram for NGC 2660. Diamonds are pulsating stars. Squares are eclipsing binaries or other types of variables.

The important results are the confirmation of the presence of five nonambiguous $\delta$ Scuti stars, all of them clearly multiperiodic, and the determination of the nature as well as the period $1.46016 \mathrm{~d}$ of the detached EB V25.

A few comments and a figure follows for the main variables in the list. 
Table 1: The variables detected in NGC 2660. Column 1: The variable names are defined here. Column 2: ID from WEBDA (Paunzen \& Mermilliod 2006), Columns 3 and 4: Coordinates, Columns 5-6: magnitude and colour.

\begin{tabular}{lllllll}
\hline ID & WEBDA & $\alpha_{2000}$ & \multicolumn{2}{l}{$\delta_{2000}$} & $\mathrm{~V}$ & $\mathrm{~B}-\mathrm{V}$ \\
& & & & & & \\
\hline V14 & & 84251.75 & -471143.1 & 17.57 & 1.15 \\
V16 & 552 & 84241.96 & -471209.0 & 14.30 & 0.66 \\
V17 & 637 & 84243.87 & -471231.6 & 16.38 & 0.72 \\
V18 & 289 & 84236.20 & -471129.0 & 14.81 & 0.70 \\
V19 & 672 & 84244.74 & -471301.2 & 14.68 & 0.67 \\
V20 & 395 & 84238.59 & -471206.7 & 14.81 & 0.70 \\
V21 & 174 & 84233.27 & -471134.7 & 14.63 & 0.68 \\
V22 & 323 & 84236.88 & -471227.3 & 18.86 & 1.50 \\
V23 & & 84215.59 & -471227.1 & 18.70 & 1.14 \\
V25 & 518 & 84241.23 & -471206.3 & 15.21 & 0.58 \\
V26 & & 84237.90 & -470841.2 & 18.49 & 1.35 \\
V27 & 205 & 84242.73 & -471335.5 & 18.21 & 1.58 \\
V28 & 589 & 84242.73 & -471035.5 & 17.60 & 1.07 \\
V29 & 411 & 84238.90 & -471037.0 & 18.19 & 1.06 \\
V30 & 406 & 84238.77 & -471238.4 & 15.28 & 0.69 \\
\hline
\end{tabular}

V25

The possible nature of this object was suggested already in Frandsen et al. (1989) based on only 14 datapoints. The new data gives a first precise period but the quality is still not quite sufficient for a precise determination of the stellar parameters. The 'noise' on the flat part of light curve might indicate that one of the components could be variable, but it could also have an instrumental origin.

The $\delta$ Scuti stars V16, V18, V19, V20 and V21

As easily seen the Fig. 3, 4, 5, 6 and 7 the light curves show the typical period and amplitude changes of pulsating variables with multiple close frequencies. The identified frequencies with $S / N>4$ are listed in Table 2.

\section{A contact binary}

A possible binary system of two close stars is presented by variable V14. It lies above the main sequence and is considerably fainter than V25. 
Table 2: The variables detected in NGC 2660. Column 1: The variable names are defined here. Column 2: ID from WEBDA (Paunzen \& Mermilliod 2006), Columns 3-5: Property of variability and Column 6: comments, where $\mathrm{EB}=$ Eclipsing Binary, $\mathrm{DS}=\delta$ Scuti star, $\mathrm{CM}=$ Cluster Member and $\mathrm{GD}=\gamma$ Dor star.

\begin{tabular}{|c|c|c|c|c|c|}
\hline$\overline{\text { ID }}$ & WEBDA & $\begin{array}{l}f \\
\mathrm{c} / \mathrm{d}\end{array}$ & $\overline{S / N}$ & $\begin{array}{l}a_{B} \\
\text { mmag }\end{array}$ & Notes \\
\hline V14 & & \multicolumn{3}{|c|}{$P=0.3259 \mathrm{~d}$} & $\mathrm{~EB}$ \\
\hline \multirow[t]{3}{*}{ V16 } & 552 & 5.86 & 12.9 & 52 & DS CM \\
\hline & & 6.31 & 10.8 & 44 & \\
\hline & & 10.50 & 4.2 & 17 & \\
\hline V17 & 637 & \multicolumn{3}{|c|}{$P=0.9 \mathrm{~d}$} & GD CM \\
\hline \multirow[t]{5}{*}{ V18 } & 289 & 6.48 & 5.9 & 18 & DS CM \\
\hline & & 7.22 & 5.8 & 12 & \\
\hline & & 12.31 & 5.4 & 14 & \\
\hline & & 12.51 & 5.3 & 13 & \\
\hline & & 11.71 & 4.6 & 14 & \\
\hline \multirow[t]{4}{*}{ V19 } & 672 & 10.07 & 8.0 & 18 & DS CM \\
\hline & & 11.16 & 5.9 & 14 & \\
\hline & & 10.50 & 5.4 & 12 & \\
\hline & & 4.83 & 5.1 & 13 & \\
\hline \multirow[t]{3}{*}{ V20 } & 395 & 7.53 & 6.6 & 26 & DS CM \\
\hline & & 5.91 & 6.5 & 26 & \\
\hline & & 6.19 & 4.2 & 16 & \\
\hline \multirow[t]{4}{*}{ V21 } & 174 & 12.78 & 7.5 & 23 & DS CM \\
\hline & & 6.96 & 5.7 & 15 & \\
\hline & & 13.61 & 4.6 & 14 & \\
\hline & & 7.86 & 4.2 & 11 & \\
\hline $\mathrm{V} 22$ & 323 & \multicolumn{3}{|c|}{$P=? ?$} & $\mathrm{~EB}, \mathrm{CM}$ \\
\hline $\mathrm{V} 23$ & & \multicolumn{3}{|c|}{$P=0.389 \mathrm{~d}$} & Contact EB \\
\hline $\mathrm{V} 25$ & 518 & \multicolumn{3}{|c|}{$P=1.46016 \mathrm{~d}$} & det. EB, CM \\
\hline V26 & & \multicolumn{3}{|c|}{$P=? ?$} & EB \\
\hline V27 & 205 & \multicolumn{3}{|c|}{$P=? ?$} & $\mathrm{~EB}$ \\
\hline V28 & 589 & \multicolumn{3}{|c|}{$P \sim 6 \mathrm{~d}$} & Var CM \\
\hline V29 & 411 & \multirow{2}{*}{\multicolumn{3}{|c|}{$\begin{array}{c}P=\text { several } \mathrm{d} \\
P=0.52 \mathrm{~d}\end{array}$}} & det. EB, CM \\
\hline V30 & 406 & & & & Var CM \\
\hline
\end{tabular}

\section{A possible $\gamma$ Dor star}

Variable V17 shows the long period and the location in the CM-diagram of the $\gamma$ Dor variables. 


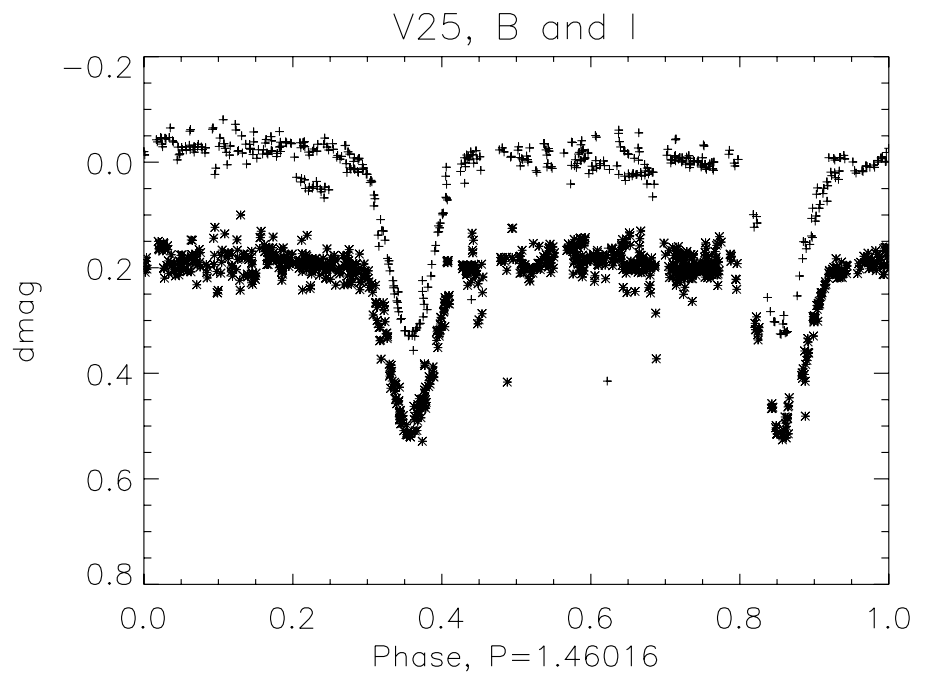

Figure 2: Phase plot for the detached EB V25. The $I$ points have been shifted by 0.2 magnitude. Differential magnitude relative to reference stars are plotted normalised to 0.0 or 0.2 outside eclipses.

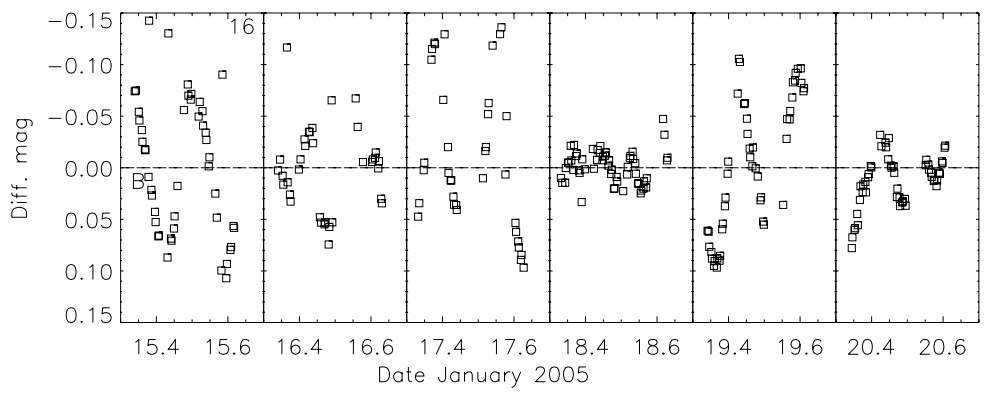

Figure 3: The $B$ light curve for $\mathrm{V} 16$. The $I$ data was of poor quality for this star due to crowding.

\section{Conclusion}

NGC 2660 is shown to be one of a small set of open clusters, where one has the potential of determining precise masses for the $\delta$ Scuti stars due to the presence of a detached eclipsing binary system not far away in mass from the pulsating 


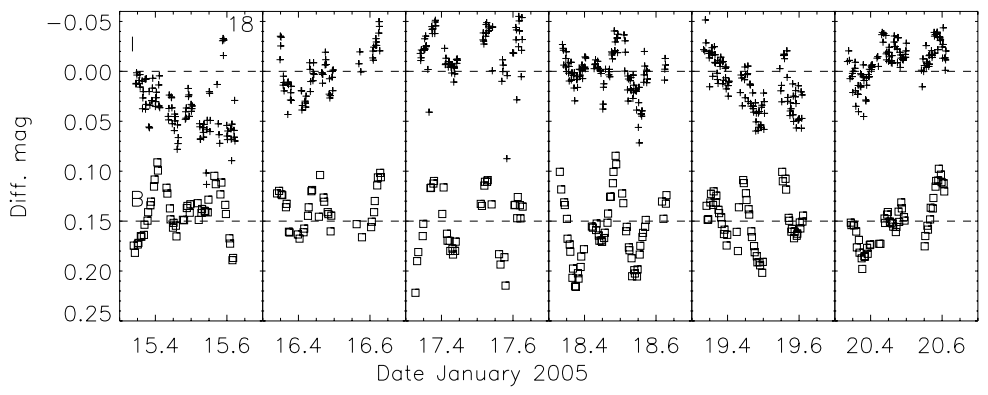

Figure 4: The $B$ and $I$ light curve for $\mathrm{V} 18$.

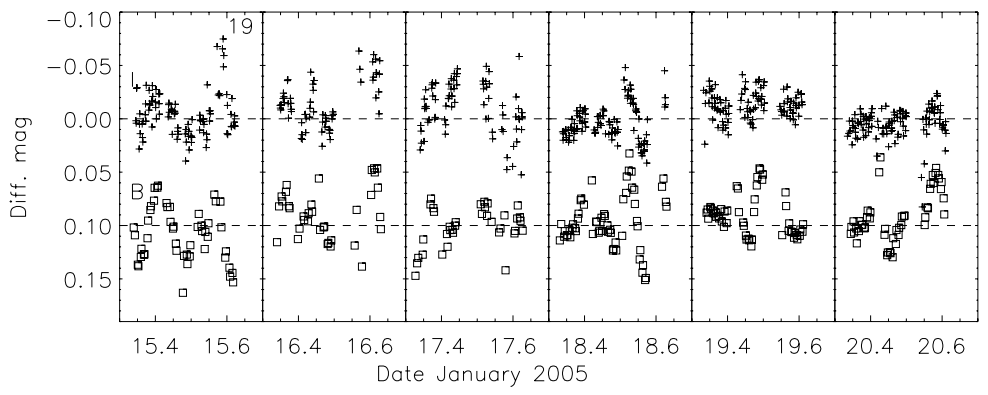

Figure 5: Same for V19.

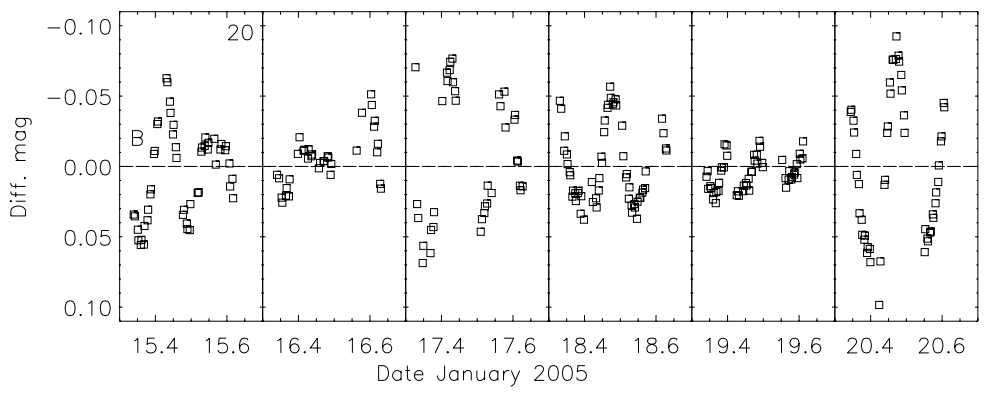

Figure 6: Same for V20, but only B is shown. 


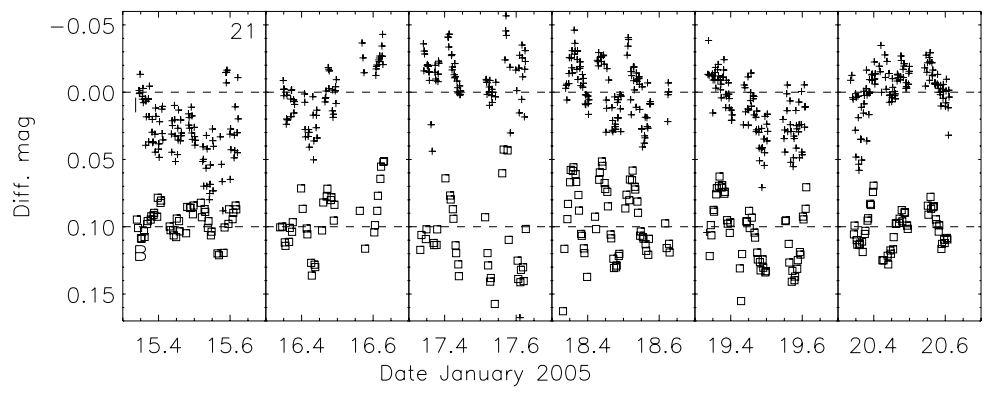

Figure 7: Same for V21.

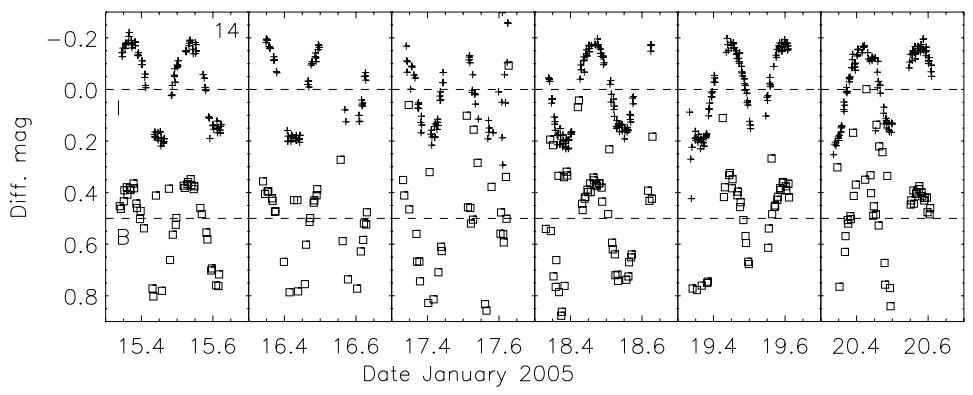

Figure 8: Contact binary V14.

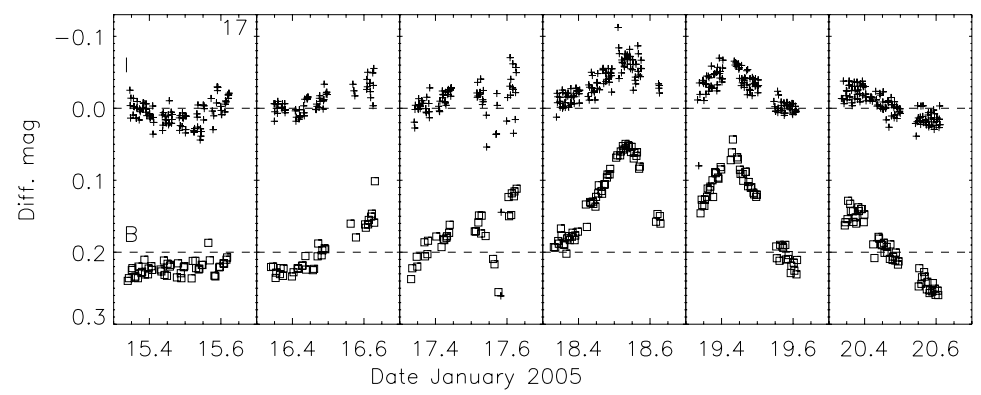

Figure 9: A $\gamma$ Dor variable: 17. 
stars. At the same time a better age determination is possible, which also is important when modeling the pulsating stars.

A small but not unimportant result is, that it is possible to find candidates for detached eclipsing binaries from a small set of frames as the confirmation of the nature of V25 shows. This knowledge is useful when searching for detached EBs in other clusters, particularly globular clusters, where detached EBs are rare but extremely important for age determinations.

Acknowledgments. Based on observations with the Danish $1.54 \mathrm{~m}$ telescope at La Silla, ESO. This research was supported by the Danish Natural Science Council through its Instrument Center for Danish Astronomy.

\section{References}

Arentoft, T., Bouzid, M. Y., Sterken, C., et al. 2005, PASP, 117, 601

Freyhammer, L. M., \& Arentoft, T., C. 2001, A\&A, 368, 580

Frandsen, S., Dreyer, P., \& Kjeldsen, H. 1989, A\&A, 215, 287

Frandsen, S., Balona, L.A., Viskum, M., et al. 1996, A\&A, 308, 132

Fox Machado, L., Pérez Hernandez, F., Suárez, J.C., et al. 2006, A\&A, 446, 611

Paunzen, E., \& Mermilliod, J.-C. 2006, http://www.univie.ac.at/webda/

Sandrelli, S., Bragaglia, A., Tosi, M., \& Marconi, G. 1999, MNRAS, 309, 739

Stetson, P.B. 1987, PASP 99, 121 